\title{
DESCRIPCIÓN DE UNA NUEVA ESPECIE DEL GÉNERO LEIODES LATREILLE, 1797 DE LA PENÍNSULA IBÉRICA (COLEOPTERA, LEIODIDAE, LEIODINAE)
}

\author{
J. A. Sáez Bolaño ${ }^{1}$ J. M. Blanco Villero² \& J. Fresneda ${ }^{3}$
}

\section{RESUMEN}

J. A. Sáez Bolaño, J. M. Blanco Villero \& J. Fresneda. 2012. Descripción de una nueva especie del género Leiodes Latreille, 1797 de la península ibérica (Coleoptera, Leiodidae, Leiodinae). Grael/sia, 68(2): 281-290.

Se describe una nueva especie de Leiodes Latreille, 1797 de la península ibérica. Los especímenes de la serie tipo proceden de Cabeza la Vaca (Sierra de Tudía, Badajoz, Extremadura). Leiodes tudiensis n. sp. pertenece al grupo de especies que presentan la quilla mesoventral baja y estriolas transversas en la superficie elitral, además de tener los metatarsómeros cónicos y el último artejo antenar claramente más estrecho que el penúltimo. Estos caracteres hacen que en el ámbito paleártico se aproxime a Leiodes brandisi (Holdhaus, 1902), L. ganglbaueri (Holdhaus, 1902), L. piliferus (Reitter, 1885), L. rugosa Stephens, 1829, L. skalitzkyi (Ganglbauer, 1899) y L. taurica (Breit, 1917). Sin embargo, la peculiar estructura general del edeago muestra semejanza únicamente con L. taurica; no obstante, hay diferencias fácilmente observables, lo cual sumado a diversos detalles adicionales de su morfología, permite separar las dos especies sin dificultad.

Palabras clave: Coleoptera; Leiodidae; Leiodinae; Leiodes; especie nueva; península ibérica; España.

\section{ABSTRACT}

J. A. Sáez Bolaño, J. M. Blanco Villero \& J. Fresneda. 2012. Description of a new species of the genus Leiodes Latreille, 1797 from the Iberian peninsula (Coleoptera, Leiodidae, Leiodinae). Grael/sia, 68(2): 281-290 (in Spanish).

In this paper a new Iberian species of Leiodes Latreille, 1797 from Cabeza la Vaca (Sierra de Tudia, Badajoz, Extremadura) is described. Leiodes tudiensis $\mathbf{n}$. sp. shares with some species of the genus the low mesoventral keel, elytral surface with grooves, last antennal article clearly narrower than the penultimate and conus-shaped metatarsi. Palearctic species of Leiodes with these characters are Leiodes brandisi (Holdhaus, 1902), L. ganglbaueri (Holdhaus, 1902), L. piliferus (Reitter, 1885), L. rugosa Stephens, 1829, L. skalitzkyi (Ganglbauer, 1899) and L. taurica (Breit, 1917). However, the structure of the aedeagus only shows similarity to $L$. taurica, although it is clearly different. The morphology of the aedeagus, together with the color pattern and other morphological details make the new species highly characteristic.

Key words: Coleoptera; Leiodidae; Leiodinae; Leiodes; new species; iberian peninsula; Spain.

Apdo. 25; 06280 Fuentes de León (Badajoz), España

Apdo. 42; 11100 San Fernando (Cádiz), España; blancovillero@gmail.com

Ca de Massa. E-25526 Llesp (Lérida), España. Museu de Ciències Naturals (Zoologia), Passeig Picasso s/n. E-08003

Barcelona, España; ffresned@gmail.com 


\section{Introducción}

El género Leiodes Latreille, 1797, de distribución casi mundial, aparece integrado en la región paleártica por 121 especies de las cuales 63 se localizan en Europa (Perreau, 2004). Tras el fundamental trabajo de Daffner (1986), únicamente han sido descritas dos especies nuevas en Europa: Leiodes graeca Svec, 1993 y L. graefi Svec, 1994; el resto fueron descubiertas en el siglo XIX y comienzos del XX. Salvo las mencionadas, el resto de especies descritas en el área paleártica son todas asiáticas.

En la península ibérica este género, así como el resto de Leiodini, ha sido poco estudiado. Fuente (1925) menciona doce especies, incluyendo todas las pirenaicas, a saber: L. brunnea (Sturm, 1807) (Ciudad Real), L. cinnamomea (Panzer, 1793) (Cuenca), L. discontignyi (Brisout, 1867) (Pirineos), L. dubia (Kugelann, 1794) (Ciudad Real, Coimbra), L. calcarata (Erichson, 1845) (= L. polita (Marsham, 1802) -Perreau, 2006-) (Altos Pirineos, Pirineos orientales, Barcelona), L. ferruginea (Fabricius, 1787) (España boreal, S. Martinho en Portugal, como L. scita), L. flavicornis (Brisout, 1883) (Ciudad Real), L. hiemalis (Abeille, 1901) (Barcelona), L. badia (Sturm, 1807) (Pirineos orientales y Ciudad Real, ésta última localización como L. similata), L. litura (Stephens, 1832) (Altos Pirineos), L. lunicollis (Rye, 1872) (Pirineos orientales) y L. nigrita (Schmidt, 1841) (León). Excluyendo las localidades exclusivamente francesas que por error incorporó Fuente (1925) -tradujo al castellano el nombre de los departamentos franceses de Hautes-Pyrénées y Pyrénées-Orientales- se obtienen nueve especies. Actualmente en el ámbito peninsular Perreau (2004) recoge diez especies: $L$. badia, L. calcarata, L. cinnamomea, L. distiguenda (Fairmaire, 1856), L. dubia (Kugelann, 1794), L. ferruginea, L. flavicornis, L. hybrida (Erichson, 1845), L. macropus (Rye, 1873) y L. nigrita. Dado que en Francia están citadas 29 especies, todo parece indicar que la península ibérica constituye un terreno casi inexplorado para este género y tribu.

Este trabajo se enmarca en el estudio más amplio que se está llevando a cabo acerca de los coleópteros de la Sierra de Tudía y cuyos resultados van apareciendo paulatinamente (Blanco \& Sáez, 2007; Blanco et al., 2007; Sáez et al., 2007; Ferrer et al., 2008; Sáez \& Blanco, 2010; Fresneda et al., 2011). Las características geológicas, climáticas y botánicas del área de estudio han sido ya descritas en Blanco \& Sáez (2007).

\section{Material y métodos}

Se han estudiado 13 ejemplares de la especie que se describe, en concreto cinco machos y ocho hembras, todos procedentes de Los Cortinales (Cabeza la Vaca, Badajoz, Extremadura, España). Para la captura sistemática de los especímenes se han utilizado trampas de caída (pitfall) colocadas a la manera descrita por Salgado et al. (2008) y cebadas con cerveza.

El estudio morfológico se efectuó sobre los ejemplares en seco mediante la observación con estereomicroscopio. Para la extracción de las genitalias de los machos fue necesaria la rehidratación de los ejemplares, siendo montadas en las mismas cartulinas con resina DMHF. Las mediciones fueron efectuadas mediante un ocular micrométrico acoplado al estereomicroscopio.

Las fotografías han sido tomadas con una cámara digital acoplada al estereomicroscopio. Los dibujos son originales de uno de los autores (J. A. Sáez).

En el estudio morfológico se han usado los términos mesoventrito, quilla mesoventral y metaventrito. En la bibliografía sobre Leiodini se había usado tradicionalmente mesosterno, quilla mesosternal y metasterno. Lawrence (1999) recupera las observaciones de Campau (1940), Ferris (1940) y Baehr (1975) -ignoradas por la gran mayoría de autores posteriores- en que se hace patente un error de observación: «... the terms mesosternum and metasternum, as used in Coleoptera, are misapplied to regions which consist of paired subcoxal elements (preepisterna), sometimes combined with paired katepisterna, the true sternum having been invaginated along the midline where the discrimen usually lies.» (Lawrence, 1999). Este autor propone los términos neutrales mesoventrito y metaventrito para reemplazar mesosterno y metasterno respectivamente. Las nuevas denominaciones se han incorporado a los manuales básicos de morfología (Beutel \& Lawrence, 2005; Lawrence et al., 2010, 2011) y su uso se va generalizando (Lawrence et al., 1999, 2011; Beutel \& Haas, 2000).

\section{Resultados}

Leiodes tudiensis n. sp. (Fig. 1)

LOCAlidad típICA. España, Badajoz, Cabeza la Vaca, Los Cortinales, 770 m, UTM (WGS84): 29 S72535 E421699.

SERIE TIPO. Holotypus: porta etiqueta de símbolo $\sigma^{7}$, más tres identificativas: «Cabeza la Vaca / Badajoz / J. Sáez leg. // Los 
Cortinales / 20-III-2011 / En TC con cerveza» [etiqueta blanca rectangular manuscrita Sáez], «Holotipo / Leiodes tudiensis n. sp. / Sáez, Blanco \& Fresneda det. 2012» [etiqueta roja rectangular, impresa], «MNCN Cat. Tipos $\mathrm{N}^{\circ} 2231 »$ [etiqueta blanca rectangular, impresa].

Paratypi: doce ejemplares con etiqueta de sexo, $40^{\pi} \sigma^{\pi}$ y 8 우 우, identificados todos con las mismas dos etiquetas: «Cabeza la Vaca / Badajoz / J. Sáez leg. // Los Cortinales / fecha / En TC con cerveza» [etiqueta blanca rectangular manuscrita Sáez], «Paratipo / Leiodes tudiensis n. sp. / Sáez, Blanco \& Fresneda det. 2012» [etiqueta roja rectangular impresa], varian únicamente las fechas que son las siguientes: $10^{7}, 24-I-2011 ; 10^{7}, 7-I I-2011 ; 10^{7}, 20-I V-2011 ; 10^{7}, 03-I I I-$ 2012; 1 우, 12-IV-2010; 1 우, 06-V-2010; 1 우, 22-III-2011; 1 우, 11-IV-2011; 1 우, 14-IV-2011. 1 우, 02-XII-2011; 1 우, 07I-2012 y 1 우, 04-III-2012.

Tanto el holotipo como ocho paratipos $\left(20^{\pi} 0^{\pi}\right.$ y 6 우 우 $)$ quedan depositados en el Museo Nacional de Ciencias Naturales de Madrid, una pareja de paratipos en la colección José Manuel Blanco Villero (San Fernando, Cádiz, España) y otra en la colección Javier Fresneda (Llesp, Lérida, España).

Diagnosis. Leiodes tudiensis n. sp. es una especie de Leiodes de tamaño medio, entre 1,9 y 2,9 $\mathrm{mm}$, bicolor, que pertenece al grupo de especies que poseen estriolas transversas en toda la superficie de los élitros, una quilla mesoventral de perfil bajo que alcanza el reborde anterior del mesoventrito; es decir, similar al tipo C de Daffner (Daffner, 1983), los metatarsómeros subcónicos y la maza antenar más oscura que el resto con el último artejo de la misma más estrecho que el penúltimo. El edeago tiene los estilos laterales del tegmen doble de largos que el lóbulo medio y provistos cada uno de ellos de una docena de cerdas en su cara interna.

DESCRIPCIÓN DEL HOLOTIPO. La forma general (Fig. 1) es oval, algo acuminada hacia atrás; la longitud total tomada entre el borde del clípeo y el ápice elitral es de 2,90 $\mathrm{mm}$, el doble que la anchura máxima, 1,45 mm; el aspecto es muy brillante, con el dorso prácticamente lampiño y algunas sedas cortas y aisladas. La cabeza y el pronoto son de color castaño muy oscuro, prácticamente negro, salvo los ángulos posteriores que son más claros; los élitros son pajizos, salvo una banda parasutural más oscura en cada élitro que se continúa en el ápex con otra, más ancha, que recorre el margen externo. La maza antenar es más oscura que el resto, que es de color amarillento. Los palpos, las mandíbulas y las patas son de color castaño claro al igual que la parte inferior.

La cabeza es transversa, casi el triple de ancha que de larga, con una longitud (excluyendo las mandíbulas) de $0,25 \mathrm{~mm}$ y una anchura máxima (a nivel de los ojos) de 0,73 $\mathrm{mm}$. El punteado es simple, muy fino y esparcido, salvo cuatro puntos mucho más gruesos en la frente, a nivel de una línea imaginaria que uniría el borde posterior de los ojos. Estos cuatro puntos aparecen agrupados de dos en dos, de tal forma que los dos centrales están más separados que ellos con los laterales. Existe un grueso reborde clipeal que se continúa con un reborde periocular anterior, mucho más fino.

Las antenas (Fig. 2) son relativamente cortas, con una longitud total de $0,91 \mathrm{~mm}$. Los antenómeros dos a seis son aproximadamente del mismo grosor, salvo el segundo que es ligeramente más grueso que los otros. El primero, segundo y tercero son sensiblemente más largos que los tres siguientes. La maza antenar es ligeramente más larga $(0,49$ $\mathrm{mm})$ que el resto $(0,43 \mathrm{~mm})$. El último artejo es más largo que ancho y claramente más estrecho que el penúltimo; el octavo es muy corto y de forma lenticular; el noveno y el décimo son de igual anchura, de forma cuadrangular, algo más anchos que largos. Las dimensiones de los antenómeros se dan en la siguiente tabla (en $\mu \mathrm{m})$ :

$\begin{array}{lccccccccccc}\text { Artejo } & \text { I } & \text { II } & \text { III } & \text { IV } & \text { V } & \text { VI } & \text { VII } & \text { VIII } & \text { IX } & \text { X } & \text { XI } \\ \text { Longitud } & 75 & 75 & 97 & 60 & 60 & 60 & 90 & 37 & 112 & 105 & 142 \\ \text { Anchura } & 68 & 52 & 45 & 45 & 49 & 53 & 108 & 75 & 154 & 154 & 97\end{array}$

El pronoto es transverso, con una longitud de $0,73 \mathrm{~mm}$ y una anchura -máxima a nivel de la base- de 1,30 $\mathrm{mm}$. El margen anterior y los laterales están rebordeados, no así la base. El punteado es simple, muy fino y esparcido, semejante al de la cabeza. Sin embargo a nivel parabasal pueden apreciarse algunos puntos más gruesos y aislados que se hacen más frecuentes y agrupados en las cercanías de los ángulos posteriores, donde delimitan un declive. Los ángulos anteriores son redondeados y los posteriores obtusos.

El escudete es pequeño, triangular y prácticamente sin puntos.

Los élitros tienen una longitud de $1,80 \mathrm{~mm}$ por una anchura máxima de 1,45 $\mathrm{mm}$. Las estrías, en número de nueve, están indicadas por hileras de puntos gruesos bien impresos, separados entre sí por dos o tres veces su diámetro. En la primera estría los puntos se continúan con una acanaladura en sus $2 / 3$ posteriores configurando de esta forma una auténtica estría. En las interestrías se observan pequeños puntos superficiales que aparecen aislados y alineados en la mitad anterior de la interestría yuxtasutural, o forman unos surcos transversos que 

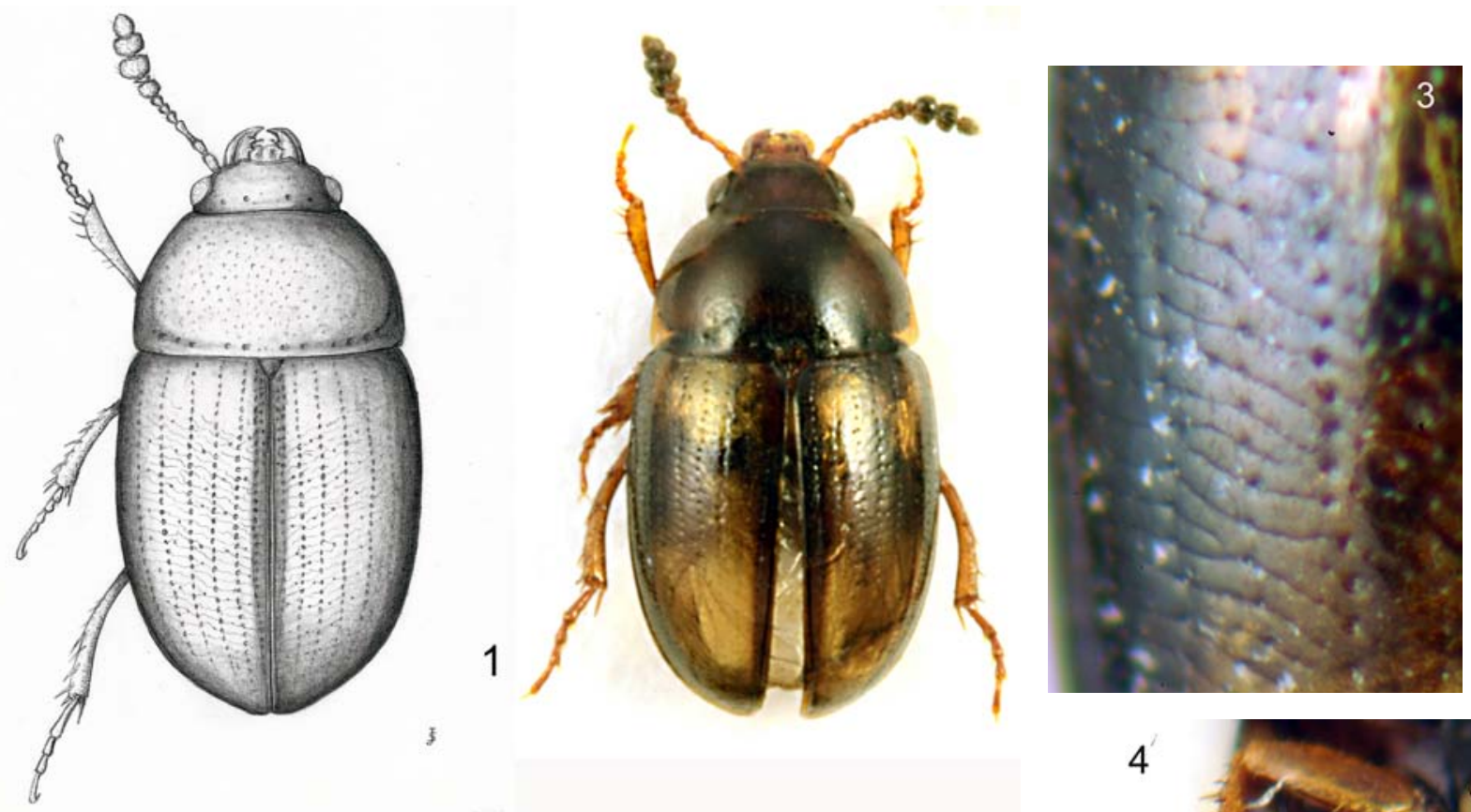

2
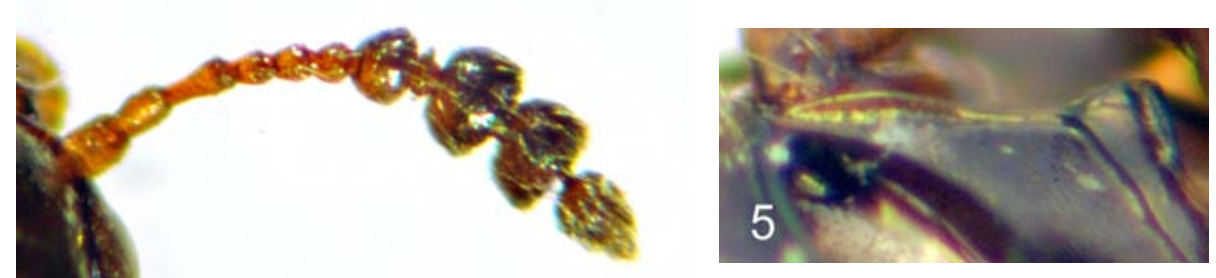

4
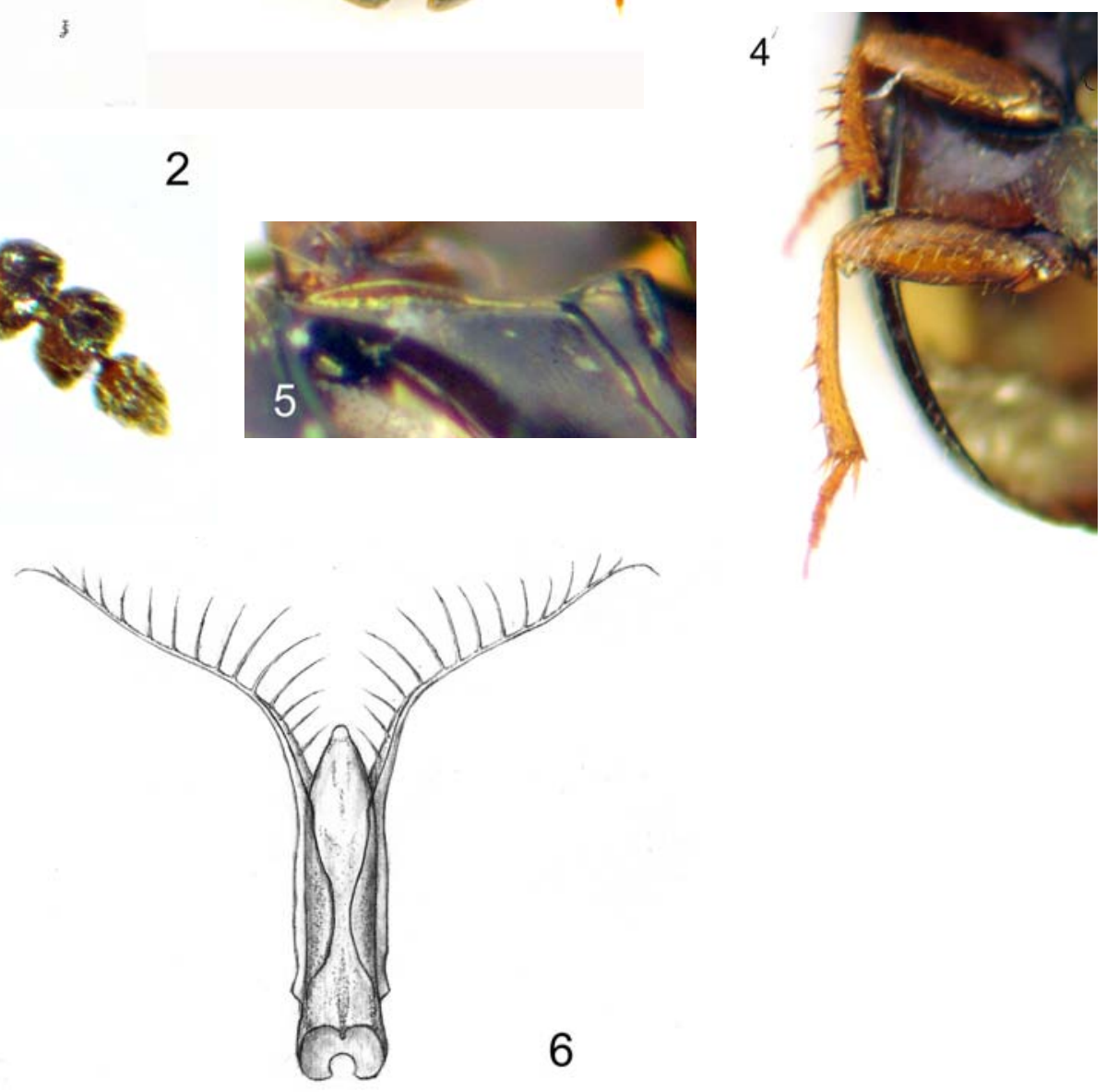

Figs. 1-6.- Leiodes tudiensis n. sp: 1) Habitus dorsal del holotipo, 2) antena, 3) estriolas elitrales, 4) metafemures, 5) quilla mesoventral, 6) edeago (paratipo).

Figs. 1-6.- Leiodes tudiensis n. sp: 1) Dorsal view of the holotype, 2) antenna, 3) elytral estriola, 4) metafemura, 5) mesoventral keel, 6) aedeagus (paratype). 
forman un ángulo de unos $25^{\circ}$ con la línea sagital en la restante superficie elitral (Fig. 3); estos surcos transversos están interrumpidos por los puntos que forman las estrías y ocasionalmente por los puntos aislados alineados de las interestrías.

Extremidades. Las tibias anteriores son relativamente finas, dos veces más anchas en el extremo distal que en el proximal, con tres fuertes espinas en la arista de la cara externa del tercio anterior; dos apicales y dos o tres más pequeñas en el tercio medio. Las tibias posteriores están ligeramente curvadas hacia adentro. Los protarsos y los mesotarsos tienen los artejos subcónicos igual que los metatarsos, aunque en estos son más alargados. Los metafémures poseen un diente agudo y ligeramente curvado hacia adentro en el extremo distal del borde posterior, donde delimita una sinuosidad. En el extremo proximal se aprecia otra sinuosidad en la vecindad del trocánter (Fig. 4). La cara inferior de los fémures tiene una pilosidad escasa, relativamente larga, integrada por sedas tumbadas hacia atrás.

Región ventral del cuerpo. El mesoventrito es glabro con arrugas discretas. La quilla mesoventral, en visión lateral, es de perfil bajo con la misma altura desde el borde anterior de las mesocoxas hasta el centro del mesoventrito donde su altura se reduce a la mitad hasta entrar en contacto con el reborde anterior del mesoventrito (similar al tipo $\mathrm{C}$ de Daffner) (Fig. 5). El metaventrito está escasamente punteado, observándose una pilosidad también escasa y rala, semejante a la de los fémures.

Edeago (Fig. 6). El lóbulo medio tiene forma de tubo de sección troncocónica que acaba en punta roma; la escotadura de la cara ventral de la base es redondeada y no muy profunda. En el saco interno se encuentran bandas de refuerzo apical, un estilete en la región media y una ancha lámina basal con el extremo apical bifurcado. Los estilos laterales del tegmen divergen y se hacen progresivamente más finos, presentando, en la cara interna de su mitad distal, once largas cerdas a modo de peine cuya longitud va en disminución hacia la punta, que termina en dos o tres cerdas terminales adicionales.

Dimorfismo SEXUAL. Las hembras se diferencian fundamentalmente por la ausencia de diente en el extremo distal del borde posterior de los metafémures, además de tener los tarsos más finos.

VARIABILIDAD. Las longitudes totales de los machos se encuentran entre 1,92 y $2,90 \mathrm{~mm}(1,92$; $1,95 ; 2,00 ; 2,05$ y 2,90$)$ y las de las hembras entre 2,10 y $2,80 \mathrm{~mm}(2,10 ; 2,45 ; 2,50 ; 2,50 ; 2,50 ; 2,60$;
2,60 y 2,80). La relación L/A se mantiene entre 1,90 y 2,00. En los ejemplares macho de mayor tamaño las metatibias son algo más curvas que en el resto y las cerdas internas de los estilos laterales del edeago varían entre 11 y 13. El patrón de coloración apenas difiere de un ejemplar a otro, únicamente una de las hembras muestra una banda pajiza continua a nivel del borde posterior del pronoto que une las manchas triangulares del mismo color de los ángulos posteriores del mismo.

Etimología. El nombre elegido deriva de la Sierra de Tudía, donde ha sido capturada. Se quiere precisar que tanto en anteriores trabajos como en este, se ha preferido el nombre geográfico de esta sierra que es efectivamente «Tudía» al de la comarca y el cerro donde radica el monasterio que reciben el nombre, más conocido, de «Tentudía». Relacionados con esta zona se han descrito un Pselaphinae (Coleoptera, Staphilinidae) endémico, Mayetia tentudiensis Outerelo \& Gamarra, 1987; una planta Compositae, Centaurea tentudaica Rivas, 1964 y un híbrido de Quercus denominado $Q$. senneniana x nothosubsp. tentudaicus Vázquez, 1995 (Fagaceae).

NotAS AUtOeCOLÓGICAS. Los Leiodinae, vulgarmente denominados «escarabajos redondos de los hongos», son en general saprófagos. Sin embargo, las especies del género Leiodes suelen excavar en el suelo en busca de ciertos hongos subterráneos, por tanto son micetófagos hipogeos (Wheeler \& Blackwell, 1984). Se han asociado a distintos tipos de hongos, por ejemplo a Polyporellus squamosus (Hudson, 1821), o el caso, mucho más conocido, del «escarabajo de la trufa», Leiodes cinnamomea (Klimaszewski \& Peck, 1986, in Dajoz, 2001). Las especies de este género parecen tener una fuerte dependencia del contenido de agua del suelo (Kühnelt, 1957); Leoides tudiensis n. sp. parece compartir esta dependencia; quizás por ello únicamente se han capturado ejemplares en los meses más húmedos del año, de enero a mayo, mes a partir del cual no ha caído ninguno en las trampas.

\section{Discusión}

El género Leiodes se caracteriza por tener el cuerpo oval y convexo, lampiño y generalmente de color uniformemente ocre o marrón más o menos oscuro; la cabeza tiene cuatro, a veces dos, gruesos puntos entre los ojos y una marcada carena en el margen anterior del clípeo; el octavo antenómero 
muestra aspecto lenticular; los élitros presentan estrías configuradas por puntos alineados o por el contrario muestran un punteado confuso; existe quilla mesoventral y la metatibia de los machos generalmente está incurvada. A partir del fundamental trabajo de Daffner (1983) las distintas tribus paleárticas de Leiodini han sido notablemente clarificadas. Hasta ese momento existió una cierta confusión incluso en el propio nombre genérico. Dado que incluso en publicaciones recientes se ha seguido usando «Liodes» y «Liodini» en vez de «Leiodes»y «Leiodini», ha sido necesaria una nueva clarificación que probablemente sea definitiva (Halliday \& Majka, 2010). Los caracteres usados por Daffner (1983) para la diferenciación de las especies del grupo pasan obligatoriamente por la forma de la quilla mesoventral. En un grupo de especies esta quilla, vista de perfil y que parte de las mesocoxas, se dirige hacia delante y, hacia la mitad de su extensión, cae abruptamente hacia el mesoventrito sin llegar a contactar con su reborde anterior, lo cual le confiere un perfil alto y aspecto triangular. Este grupo de especies fueron encuadradas en el subgénero Oosphaerula Ganglbauer, 1896, hoy sinónimo de Leiodes. El otro gran grupo de especies engloba a aquellas en las que dicha quilla mesoventral, de perfil bajo, avanza con la misma altura desde el borde anterior de las mesocoxas hacia el centro de la misma. Dentro de este grupo Daffner (1983) distingue a su vez tres subgrupos según contacte (tipo C) o no con el reborde anterior del mesoventrito $\mathrm{y}$, en este último caso, si lo hace por medio de una escotadura (tipo B) o acabando en el plano del mesoventrito (tipo A). Con posterioridad el propio Daffner (1986) redefine el tipo $\mathrm{C}$ y añade el tipo D para las quillas más elevadas. Recientemente Svec (2008) ha revisado los tipos de quilla basándose en el primer concepto de Daffner (1983), añadiendo cinco tipos adicionales que suman un total de ocho. En este trabajo las quillas de tipo bajo las clasifica de forma similar A, B, $\mathrm{C}$ o D. Este último tipo resulta de la subdivisión del primitivo B de Daffner (1983), sólo que la escotadura aparece en la misma quilla y no en el propio mesoventrito. Las quillas altas las subdivide en tres tipos con las letras griegas alfa, beta y delta.

Otro carácter fundamental para distinguir grupos de especies es si el punteado elitral se alinea formando pseudoestrías o, por el contrario es irregular y confuso. Otro carácter discriminatorio consiste en la presencia o ausencia de estriolas elitrales transversas que pueden aparecer en toda la superficie elitral o únicamente a los lados. Otros caracteres también importantes son la forma de los ángulos posteriores del pronoto y la anchura del último artejo antenar en relación con el penúltimo.

Atendiendo a todos estos caracteres, Leiodes tudiensis $\mathrm{n}$. sp. puede agruparse únicamente con $L$. brandisi (Holdhaus, 1902), L. ganglbaueri (Holdhaus, 1902), L. piliferus (Reitter, 1885), L. rugosus Stephens, 1829, L. skalitzkyi (Ganglbauer, 1899) y L. taurica Breit, 1917. De L. rugosus se diferencia porque esta especie es mayor $(3,20-4,50$ $\mathrm{mm}$ ) y tiene los ángulos posteriores del pronoto redondeados; L. skalitzkyi tiene los artejos de los metatarsos también cónicos pero su edeago responde a otro modelo (Daffner, 1983: fig. 140); L. brandisi tiene el último artejo antenar casi tan ancho como el penúltimo; L. ganglbaueri y L. piliferus tienen las antenas de color uniformemente castaño; además $L$. piliferus tiene una quilla mesoventral de tipo «A» de Daffner (1983).

Sin embargo, si atendemos a la estructura general del edeago, la única especie semejante es $L$. taurica. Este tipo de edeago se caracteriza por poseer dos largos estilos laterales, el doble de largos que el lóbulo medio, y provistos de una serie de cerdas en su margen interno a modo de peine cuyas longitudes disminuyen hacía el ápice. Es un modelo de edeago peculiar dentro del género. Tanto en el edeago como en la morfología externa se observan las siguientes diferencias:

- L. tudiensis n. sp. Edeago (Fig. 6): en los estilos laterales del tegmen a nivel del ápex del lóbulo medio no existe sinuosidad y el número de cerdas es de once a trece; la escotadura de la base del lóbulo medio es poco profunda y redondeada. Morfología externa (Fig. 7A): es una especie bicolor; las antenas también son bicolores pues tienen la maza oscura; el séptimo antenómero es transverso y el noveno y décimo son subcuadrados; los metatarsómeros son subcónicos; las metatibias del macho están débilmente incurvadas; el punteado de las series elitrales es más grueso.

- L. taurica. Edeago (Fig. 8; Daffner, 1983: fig. 150): en los estilos laterales del tegmen a nivel del ápex del lóbulo medio existe sinuosidad y el número de cerdas es del doble que en la otra especie; la escotadura de la base del lóbulo medio es muy profunda y alargada. Morfología 

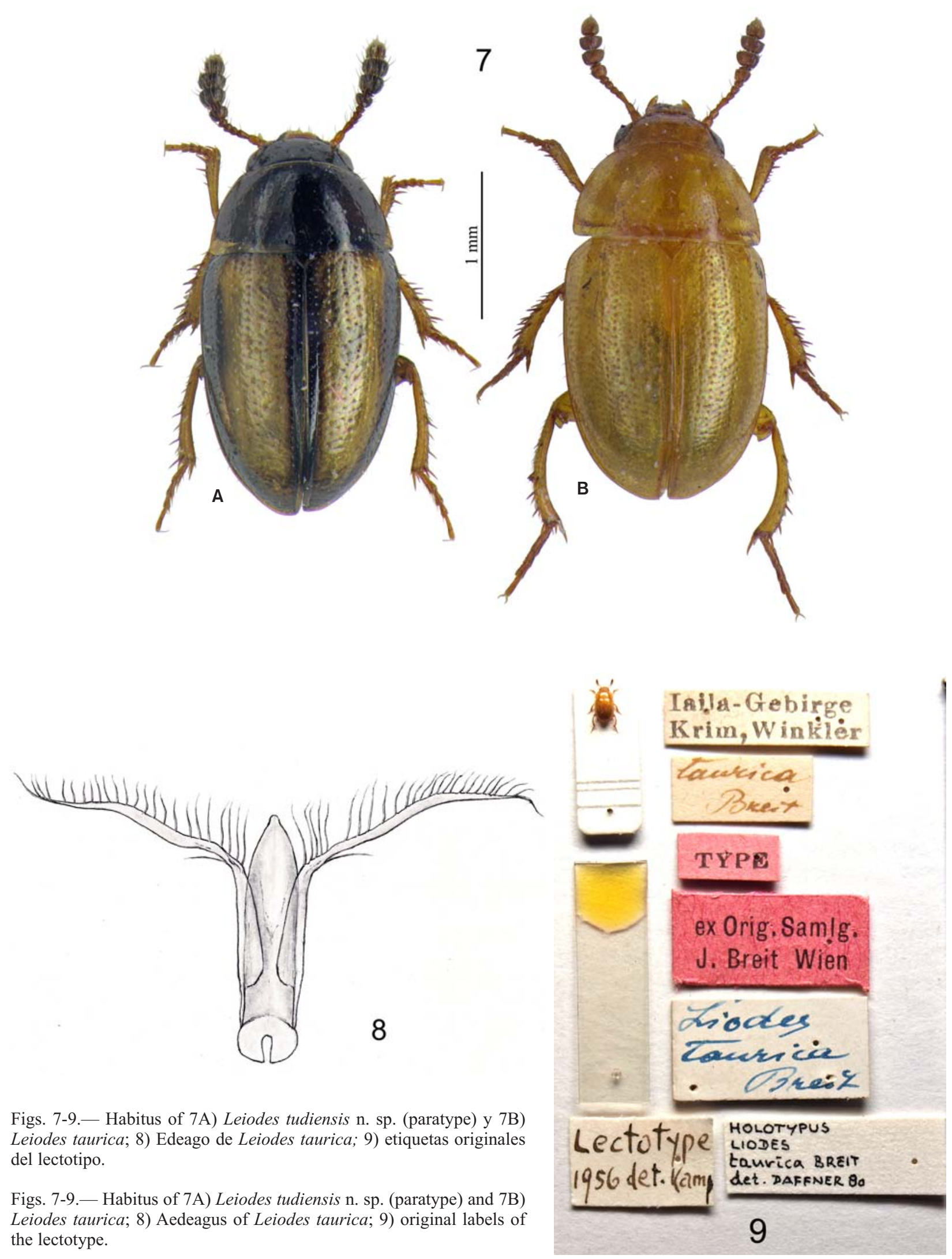
externa (Fig. 7B): la coloración es uniformemente ocre; las antenas son uniformemente amarillentas, incluida la maza; el séptimo antenómero es subcuadrado y el noveno y el décimo son fuertemente transversos; los metatarsómeros son subcilíndricos; las metatibias del macho están fuertemente incurvadas; el punteado de las series elitrales es más fino.

Biogeografía. Leiodes taurica se conoce únicamente por la serie típica que está integrada por seis ejemplares (Breit, 1917) a los que se suman dos más (Daffner, 1983); todos ellos proceden de la localidad típica, Jaila-Gebirge, que está situada en una zona montañosa del sur de la península de Crimea (Ucrania). La distribución del conjunto formado por Leiodes tudiensis n. sp. y L. taurica es un verdadero modelo de disyunción Irano-Turánica: dos especies vicariantes con distribuciones esteoeste. Este tipo de distribución con presencia en los relieves del Mar Negro en su extremo oriental, y en los relieves del Mediterráneo occidental se puede explicar por «continuidad de especies relictas» o por «colonización secundaria» (Ribera \& BlascoZumeta, 1998); estos autores encuentran en Los Monegros (Aragón, España) 63 especies con ese mismo tipo de distribución disyunta, pertenecientes a 10 órdenes y 37 familias. La existencia de las mismas relaciones biogeográficas en tan elevado número de especies, a las que ahora hay que añadir Leiodes tudiensis n. sp. y L. taurica, parece apoyar la hipótesis de su continuidad; en consecuencia se podrían considerar relictos de una fauna transPaleártica de finales del Cenozoico.

\section{Designación del lectotipo de Liodes taurica Breit, 1917}

Breit (1917) no designa ningún ejemplar tipo portanombre sino que se limita a indicar el número, sexo y recolector de los ejemplares estudiados: «Mir liegen $30^{\prime} \sigma^{\prime \prime}$ und 3 우 9 dieser Art vor, welche meine Freunde Emil Moczarski und Albert Winkler im Jaila-Gebirge in der Krim durch Aussieben feuchten Laubes entdeckten.»; por lo tanto hay que considerarlos todos sintipos. Estos ejemplares se han localizado en la colección George Frey de Munich (actualmente depositada en el Naturhistorisches Museum Basel, Suiza). Con posterioridad alguien añadió a uno de los machos la etiqueta «Lectotype / 1956 det. Kamp» sin proceder a su publicación y más tarde Daffner (1983) indica erróneamente que existe un holotipo y así lo etiqueta. Para la descripción del nuevo taxón que se describe en este artículo resulta necesaria la caracterización precisa del modelo que representa el nombre L. taurica; por este motivo se designa su lectotipo; este acto taxonómico contribuye a la estabilidad de la nomenclatura zoológica.

LeCTOTYPUS DE LIODES TAURICA BReIT (a partir de la presente designación) (Fig. 9). Un macho etiquetado: «Iaila-Gebirge / Krim, Winkler» [etiqueta blanca rectangular impresa], «taurica / Breit» [etiqueta blanca rectangular manuscrita Breit], «TYPE» [etiqueta roja rectangular impresa], «ex. Orig. Samlg. / J. Breit Wien» [etiqueta roja rectangular impresa], «Liodes / taurica /Breit» [etiqueta blanca rectangular manuscrita], «Lectotype / 1956 det. Kamp» [manuscrita], «HOLOTYPUS / LIODES / taurica BREIT / det. DAFFNER 80» [etiqueta blanca rectangular manuscrita Daffner], etiqueta de papel de acetato con el edeago montado en bálsamo del Canadá; se ha añadido la etiqueta roja rectangular impresa: «LeCtotypus / Liodes taurica Breit / Sáez, Blanco \& Fresneda des. 2012».

Paralectotypi de Liodes taURICA Breit (20 $0^{7} \sigma^{x}$ y 3 우 웅. Un macho y una hembra etiquetados: «Iaila-Gebirge / Krim, Winkler» [etiqueta blanca rectangular impresa], «taurica / Breit» [etiqueta blanca rectangular manuscrita Breit], «TYPE» [etiqueta roja rectangular impresa], «ex. Orig. Samlg. / J. Breit Wien» [etiqueta roja rectangular impresa], «Paratypoid» [etiqueta blanca rectangular manuscrita]. El macho incorpora una etiqueta de papel de acetato con el edeago montado en bálsamo del Canadá. Las otras dos hembras están etiquetadas de igual forma pero incorporan además la etiqueta «PARATYPUS / LIODES / taurica BREIT / det. DAFFNER 80» [etiqueta blanca rectangular manuscrita Daffner] y una de las dos no lleva la etiqueta escrita por Breit «taurica / Breit». Un macho etiquetado: «Iaila-Gebirge / Krim, Moczarski» [etiqueta blanca rectangular impresa], «taurica / Breit» [etiqueta blanca rectangular manuscrita Breit], «TYPE» [etiqueta roja rectangular impresa], «ex. Orig. Samlg. / J. Breit Wien» [etiqueta roja rectangular impresa], «Paratypoid» [etiqueta blanca rectangular manuscrita], «PARATYPUS / LIODES / taurica BREIT / det. DAFFNER 80» [etiqueta blanca rectangular manus- 
crita Daffner]. A los cinco ejemplares se les ha añadido una etiqueta roja rectangular impresa: «PARalectotypus / Liodes taurica Breit / Sáez, Blanco \& Fresneda des. 2012».

\section{Agradecimientos}

A Z. Svec (Praga, República Checa) especialista en Leiodini, por su ayuda en el estudio de la nueva especie que se describe. También a M. Brancucci y E. Sprecher, conservadores del Naturhistorisches Museum Basel (Suiza) por el préstamo de la serie tipo de Leiodes taurica y a I. Ribera (Barcelona, España) por su corrección de los textos en inglés.

\section{Referencias}

Baehr, M., 1975. Skelett und Muskulatur des Thorax von Priacma serrata LeConte (Coleoptera, Cupedidae). Zeitschrift für Morphologie der Tiere, 81: 55-101.

Beutel, R. G. \& Haas, F., 2000. Phylogenetic Relationships of the Suborders of Coleoptera (Insecta). Cladistics, 16: 103-141.

Beutel, R. G. \& Lawrence, J. F., 2005. 4. Coleoptera, Morphology. Handbook of Zoology. Vol. IV. Arthropoda: Insecta. Part 38 Coleoptera, Beetles. Vol. 1: Morphology and Systematics. R. G. Beutel \& R. A. B. Leschen (eds.). Walter de Gruyter. Berlin: 23-27.

Blanco Villero, J. M. \& Sáez Bolaño, J. A., 2007. Scarabaeoidea (Coleoptera) de la Sierra de Tudía (Badajoz, Extremadura, España): I. Familia Lucanidae. Boletín de la Sociedad Entomológica Aragonesa, 40: 351-358.

Blanco Villero, J. M., Sáez Bolaño, J. A., Bahillo, P. \& López-Colón, J. I., 2007. Scarabaeoidea (Coleoptera) de la Sierra de Tudía (Badajoz, Extremadura, España): II. Inventario preliminar de especies. Boletín de la Sociedad Entomológica Aragonesa, 41: 347-357.

Breit, J., 1917. Beiträge zur Kenntnis der europäischen Käferfauna. Koleopterologische Rundschau, 6: 6873.

Campau, E. J., 1940. The morphology of Chauliognathus pennsylvanicus (De Geer) (Coleoptera: Cantharidae). Microentomology, 5: 57-86.

Daffner, H., 1983. Revision der paläarktischen Arten der Tribus Leiodini Leach (Coleoptera, Leiodidae). Folia Entomologica Hungarica, 44(2): 9-163.

Daffner, H., 1986. Die Arten der Gattung Leiodes Latreille (Coleoptera, Leiodidae) aus Indien und Nepal. Revue Suisse de Zoologie, 93: 71-87.

Dajoz, R., 2001. Entomología forestal. Los insectos y el bosque. Madrid. 548 pp.
Ferrer Andreu, J. de, Sáez Bolaño, J. A. \& Blanco Villero, J. M., 2008. Los Histeridae (Coleoptera) de la Sierra de Tudía (Badajoz, Extremadura, España). Boletín de la Sociedad Entomológica Aragonesa, 43: 409-415.

Ferris, G. F., 1940. The myth of the thoracic sternites of insects. Microentomology, 5: 87-90.

Fresneda, J., Sáez Bolaño, J. A. \& Blanco Villero, J. M. 2011. Los «Hydradephaga» (Coleoptera: Dytiscidae, Gyrinidae, Haliplidae, Noteridae, Paleobiidae) de Badajoz, España. Boletín de la Sociedad Entomológica Aragonesa, 49: 211-216.

Fuente, J. M. de la, 1925. Catálogo sistemático-geográfico de los Coleópteros observados en la Península Ibérica, Pirineos propiamente dichos y Baleares. Boletín de la Sociedad Entomológica de España, 8(2): 41-43.

Halliday, R. B. \& Majka, C. G., 2010. Clarification of the status of the genus names Leiodes Latreille 1796 (Coleoptera), Liodes von Heyden 1826 (Acari) and Neoliodes Berlese 1888 (Acari). Zootaxa, 2600: 6165.

Kühnelt, W., 1957. Biología del suelo. CSIC. Madrid. 267 pp.

Lawrence, J. F., 1999. The Australian Ommatidae (Coleoptera: Archostemata): new species, larva and discussion of relationships. Invertebrate Taxonomy, 13: 369-390.

Lawrence, J. F., Beutel, R. G., Leschen, R. A. B. \& Slipinski, A., 2010. 2. Glossary of Morphological Terms. Handbook of Zoology. Arthropoda: Insecta. Coleoptera, Beetles. Vol. 2: Morphology and Systematics. R.A.B. Leschen, R.G. Beutel \& J. F. Lawrence (eds.). Walter de Gruyter. Berlin: 9-20.

Lawrence, J. F., Hastings, A. M., Dallwitz, M. J., Paine, T. A. \& Zurcher, E. J., 1999. Beetles of the World: A Key and Information System for Families and Subfamilies. CD-ROM, Version 1.0 for MSWindows. CSIRO Publishing, Melbourne.

Lawrence, J. F., Slipinski, A., Seago, A. E., Thayer, M. K., Newton, A. F. \& Marvaldi, A. E., 2011. Phylogeny of the Coleoptera based on morphological characters of adults and larvae. Annales Zoologici, 61(1): 1-217.

Perreau, M., 2004. Family Leiodidae (pp. 133-203): in LÖBL, I. \& A. SMETANA (editors): Catalogue of Paleartic Coleoptera. Vol. 2. Apollo Books. Stenstrup. 942 pp.

Perreau, M., 2006. Errata for Volume 2. In: I. Löbl, I. \& A. Smetana (eds.). Catalogue of Palaeartic Coleoptera. Vol. 3. Apollo Books. Stenstrup: 23.

Ribera, I. \& Blasco-Zumeta, J., 1998. Biogeographical links between steppe insects in the Monegros region (Aragón, NE Spain), the eastern Mediterranean, and central Asia. Journal of Biogeography, 25: 969-986. 
Sáez Bolaño, J. \& Blanco Villero, J. M., 2010. Los colévidos epigeos (Coleoptera, Leiodidae, Cholevinae) de la Sierra de Tudía (Badajoz, Extremadura, España). Boletín de la Sociedad Entomológica Aragonesa, 47: 357-361.

Sáez Bolaño, J., Blanco Villero, J. M., Bahillo de la Puebla, P. \& López-Colón, J. I., 2007. Cleroidea de la Sierra de Tudía (Badajoz, Extremadura, España). I. Familias Cleridae Latreille, 1802, Prionoceridae Lacordaire, 1857 y Malachiidae Fleming, 1821 (Coleoptera, Cleroidea). Boletín de la Sociedad Entomológica Aragonesa, 41: 407-412.

Salgado, J. M., Blas, M. \& Fresneda, J., 2008. Coleoptera, Cholevidae. En: Fauna Ibérica. Vol. 31. Ramos, M. A. et al. (eds.). Museo Nacional de Ciencias Naturales, CSIC. Madrid. 799 pp.

Svec, Z., 2008. New Chinese and Nepalese Leiodes Latreille (Coleoptera: Leiodidae: Leiodinae). Studies and reports of District Museum Prague-East. Taxonomical Series, 4(1-2): 241-258.
Wheeler, Q. \& Blackwell, M., 1984. Fungus-insect relationships: perspectives in ecology and evolution. Columbia University Press. 514 pp. 